\title{
UWB Wearable Textile Antenna
}

\author{
Mai A. R. Osman ${ }^{a}$, M. K. A. Rahim ${ }^{a^{*}}$, N. A. Samsuri ${ }^{a}$, M. K. Elbasheer ${ }^{a}$, M. E. Ali ${ }^{a}$ \\ ${ }^{a}$ Faculty of Electrical Engineering, Universiti Teknologi Malaysia, 81310 UTM Johor Bahru \\ *Corresponding author: mkamal@fke.utm.my
}

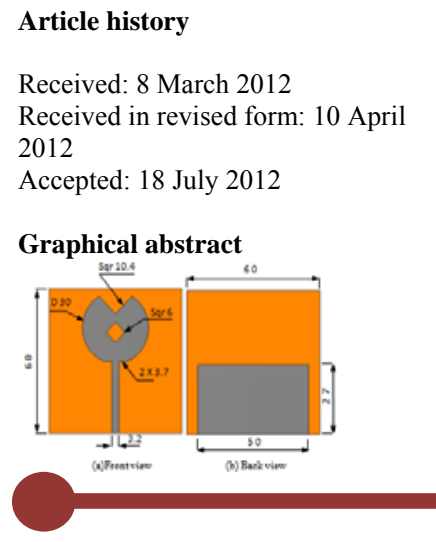

1.0 INTRODUCTION

The steadily progressing miniaturization in microelectronics along with other new technologies enables wearable computing to integrate functionality in clothing. Thus, integration in textiles ideally combines the requirements of wearable computing and wireless body area networks (WBANs), since clothing is unobtrusive and offers large area of close proximity to human body. With antennas playing a decisive role in wireless wearable systems, utilization of textile materials for the development of flexible wearable antenna has emerged aiming the robustness, flexibility, and satisfactory of wireless wearable devices. On the other hand, Ultra Wideband (UWB) transmission device does not need to transmit high-power signal to the receiver and can have a longer battery life or be smaller to reduce the wearable devices size [1-3]. In addition, UWB is a high data rate technology with large bandwidth signals that provide robustness to jamming [4, 5].

Several papers have been published about the design, fabrication and applications of wearable antennas and systems [6, 7]. Other researchers have taken quantum leaps in utilizing textile materials as antenna substrate $[8,9]$. Wearable antennas have been developed in the form of flexible metal patches on textile substrates [10], while a dual band wearable antennas was discussed in details in $[11,12]$. With all these mentioned findings, markets opened up to a wide range of potential requirements and investigations for these novel materials considering UWB antenna designs and applications. Consistent with all these facts mentioned above, authors of the current manuscript aimed to make closer steps towards real wearability by merging the UWB technology with textile technology. Therefore, UWB antennas using clothing materials as antenna substrates are fabricated and presented in this paper. Hence, substrate materials investigations involved two fabrics; flannel and jeans, while the radiating element and ground plane are made from copper self-adhesive conducting sheet. The following sections shall discuss in more details about the specifications of materials used, the proposed antenna designs as well as comparisons between simulated and measured results.

\subsection{WEARABLE ANTENNA MATERIALS}

For flexible antennas, textile materials form interesting substrates, because fabric antennas can be easily integrated into clothes. Textile materials generally have a very low dielectric constant, which reduces the surface wave losses and improves the impedance bandwidth of the antenna. In this paper, planer monopole UWB antennas have been designed and analyzed. Two types of fabrics (flannel and jeans) are considered for investigations in order to study their aptness to be used as substrate materials. Both proposed fabric materials are 100\% cotton materials with smooth and firm surface features, while the thickness of each fabric is $1 \mathrm{~mm}$. However, in order to model a fabric, it is important to know its relative permittivity. Thus, the measured relative permittivity at several frequencies for flannel and jeans fabrics was almost 1.7 with loss tangent of 0.025 . Conductive parts are made out of copper tape with a thickness of $0.03 \mathrm{~mm}$. These mentioned features together made the antenna flexible in nature.

\subsection{DESIGN CONSIDERATION}

In general, the design and construction of the UWB planer monopole antenna consist of a circular parch to be placed at the top of fabric substrate material; a microstrip feed line and a partial 
ground plane beneath the fabric substrate material. At the starting point in designing UWB fabric antenna, the main patch is initially calculated according to [13-15] and using Equation 1 in order to create circular patch at the centre frequency of the UWB spectrum of $7.85 \mathrm{GHz}$. Where $a$ is the radius of the circular patch antenna in millimetre, $f_{r}$ is the resonance frequency in $\mathrm{GHz}$, and $\varepsilon_{r}$ is the relative permittivity of the textile substrate material.

$$
a=\frac{87.94}{f r \sqrt{\varepsilon_{r}}}
$$

The copper conducting sheet with a thickness of $0.03 \mathrm{~mm}$ is proposed to be used as the conducting parts of this UWB antenna design. Since both fabrics have the same thickness with similar dielectric properties, simulation process of UWB fabric antenna designs using flannel and jeans fabrics are combined in this section in order to avoid replication. Thus, simulation process is conducted considering flannel fabric with a thickness of $1 \mathrm{~mm}$ and dielectric constant of 1.7 for the planer monopole UWB antenna design. In addition, the transmission line feeding technique is introduced in order to connect the main radiating circular patch to the $50 \Omega \mathrm{Sub}$ Miniature Version (SMA) connector where the position is determined according to [16, 17]. Moreover, a partial ground plane was implemented for the proposed antenna design; hence, such types of truncated ground plane play an important role in broadband and wideband characteristics of the designed antennas. In particular, the process of ground plane truncation acts as an impedance matching element that controls the impedance bandwidth of the circular patch. Thus, ground plane was truncated by slicing the top portion of the ground plane that covers the radiating patch element to less than half and covers the transmission line section only. For further optimizations, one slit at the top of the circular patch and two slits at the bottom of both sides of the main radiating patch are introduced. In addition, a rotated square hole (slot) at the centre of the circular patch is also needed to fine-tune the impedance bandwidth at certain frequencies. Therefore, with the incorporation of these slits and even the centre slot, the impedance bandwidth throughout the spectrum of the UWB antenna frequencies is well matched below the level of 10-dB.

Figure 1 shows the CST simulation software model that has been conducted in air space along with dimensions in millimetre of the proposed antenna design using flannel fabric as substrate material. After optimization, the substrate dimensions are set to 60 $\mathrm{mm} \times 60 \mathrm{~mm}$ with a patch radius of $15 \mathrm{~mm}$.

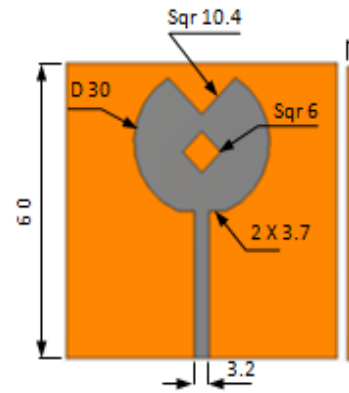

(a)Front view

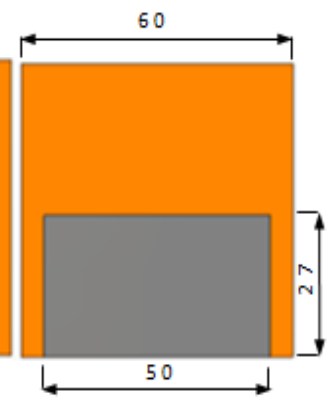

(b) Back view
Figure 1 CST simulation software model and dimensions in millimeter of the optimized UWB planer monopole antenna design using flannel fabric substrate material

\subsection{FABRICATION AND MEASUREMENT}

Fabrication process of the wearable textile UWB antennas was conducted using copper conducting sheet, which was cut using a simple cutting tool (scalpel). Moreover, the copper conducting sheet is backed by self-adhesive that ease the process of attachment by pressing it easily and nicely over the surface of fabric substrate materials. Therefore, copper conducting sheet turned out to be good solutions as it has been evenly placed and fastened over fabric substrate materials without any detaches.

\subsection{Simlated and Measured Reflection Coefficient $\left(S||_{11} \mid\right)$ Results}

A network analyser is used to measure the reflection coefficient $\left(\left.\mathrm{S}\right|_{11} \mid\right)$ results of the fabricated UWB antenna prototypes. Figure 2 (a) demonstrates a snapshot of the fabricated prototype using flannel fabric, while Figure 2 (b) shows a snapshot of the fabricated prototype using jeans fabric. In addition, $\left(\left|S_{11}\right|\right)$ measurement environment of UWB antenna prototype using flannel and jeans fabrics are illustrated in Figure 3 (a) and (b) respectively.

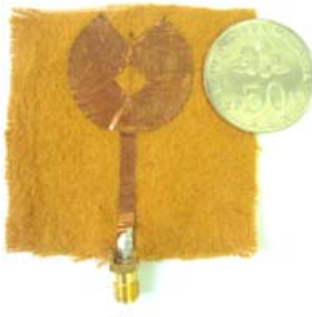

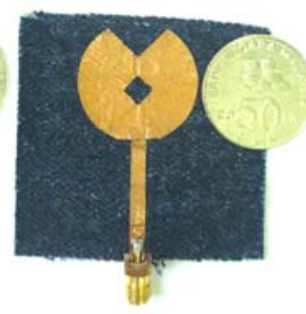

Figure 2 Snapshots of fabricated UWB antenna prototypes using, (a) flannel fabric, (b) Jeans fabric

Moreover, Figure 4 made an apparent verification and comparison between simulated and measured $\left(\left|\mathrm{S}_{11}\right|\right)$ results of the proposed design considering the usage of flannel and jeans fabrics as substrate materials. The solid line demonstrates the simulated $\left(\left|S_{11}\right|\right)$ and bandwidth results of the UWB antenna design, while the dotted line shows the measured $\left(\left|\mathrm{S}_{11}\right|\right)$ and bandwidth results of flannel UWB antenna prototype, and the long dashed line illustrates the measured $\left(\left|\mathrm{S}_{11}\right|\right)$ and bandwidth results of jeans UWB antenna prototype.

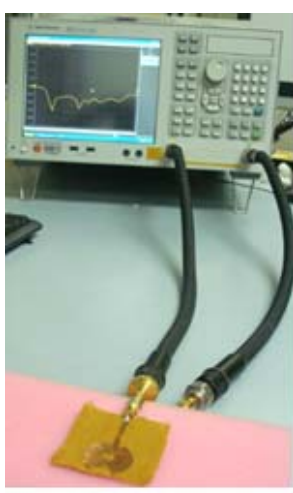

(a)

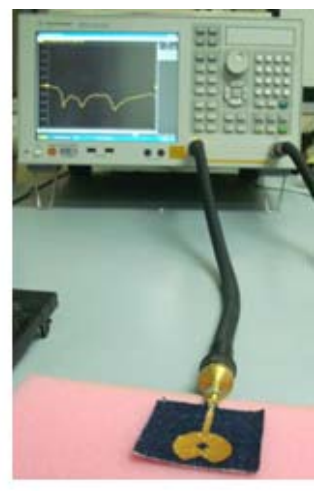

(b)
Figure $3 \quad\left(\left|\mathrm{~S}_{11}\right|\right)$ measurement environment of both UWB antennas prototypes considering (a) One layer of jeans fabric, and (b) Three stacked layers of jeans fabric 
UWB Fabric Antenna

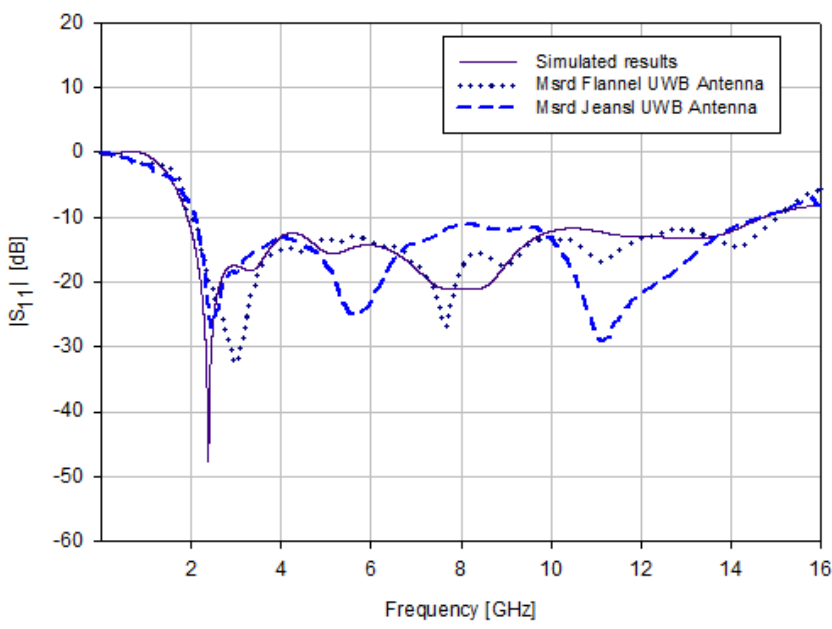

Figure 4 Comparisons of $\left(\left|\mathrm{S}_{11}\right|\right)$ simulated and measured results of UWB antenna design, (a) _ Simulated results, (b) . . . Flannel measured results, (c) _ - Jeans measured results

$\left(\left|\mathrm{S}_{11}\right|\right)$ results showed that UWB fabric antenna designs produced good impedance matching with reflection coefficient results being below the level of $10-\mathrm{dB}$ in all cases. The bandwidth expands from the lower frequency of $2 \mathrm{GHz}$ until the upper frequency of $15 \mathrm{GHz}$. Simulated and measured results achieved an Absolute Band-Width (ABW) of more than $13 \mathrm{GHz}$, which is counterpart to a BW of $7.5 \%$ over the intended UWB frequency range. Although the slight deviations between simulated and measured results are visible to some extent, Figure 4 exhibited that $\left(\left|\mathrm{S}_{11}\right|\right)$ results were comparable with an acceptable and reasonable shift on measured results occurred due to fabrication and measurement tolerances. Results indicated that the measured dielectric properties values of both fabrics were valid enough to provide design information, since the targeted UWB frequency range is reasonably well met and hence produced comparable measured results.

\subsection{Simulated Current Distribution Results}

Current distribution results make the idea visible in order to understand how the current flows on the conducting areas of the simulated UWB textile antenna using flannel fabric as the substrate material along with the copper sheet as the conducting material. Figure 5 demonstrates six frequency samples of current flow simulated results at $3 \mathrm{GHz}, 5 \mathrm{GHz}, 8 \mathrm{GHz}, 10 \mathrm{GHz}, 13 \mathrm{GHz}$ and $15 \mathrm{GHz}$ for the proposed antenna design. From Figure 5 (a), (b) and(c), high strength of current in the range of frequencies from 2 $\mathrm{GHz}$ up to $8 \mathrm{GHz}$ can be recognized along the conducting parts of the antenna and especially along the transmission line as well as the bottom and the top of the radiating patch. However, the rest of frequencies from $9 \mathrm{GHz}$ up to $15 \mathrm{GHz}$ were found to have high strength of current along the transmission line as well as at the bottom of the circular patch, as illustrated in Figure 5 (d), (e) and (f).

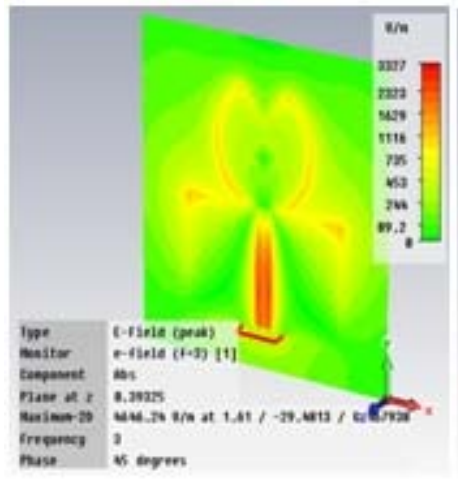

(a) $3 \mathrm{GHz}$

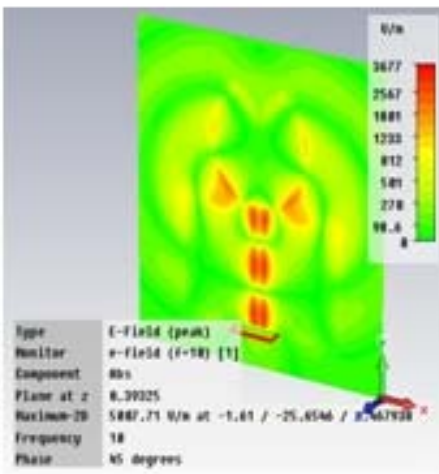

(d) $10 \mathrm{GHz}$

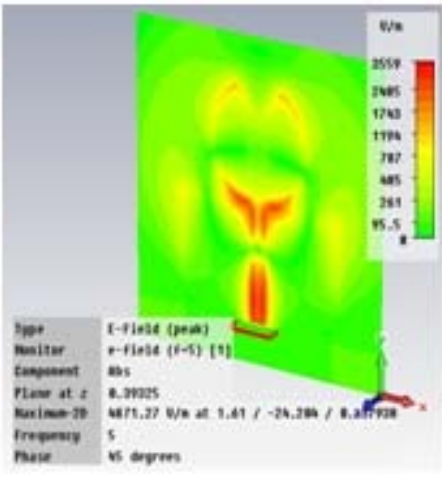

(b) $5 \mathrm{GHz}$

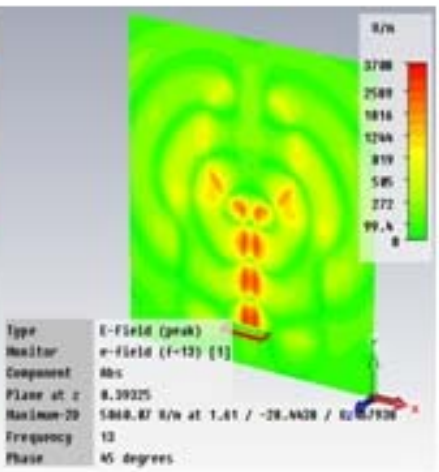

(e) $13 \mathrm{GHz}$

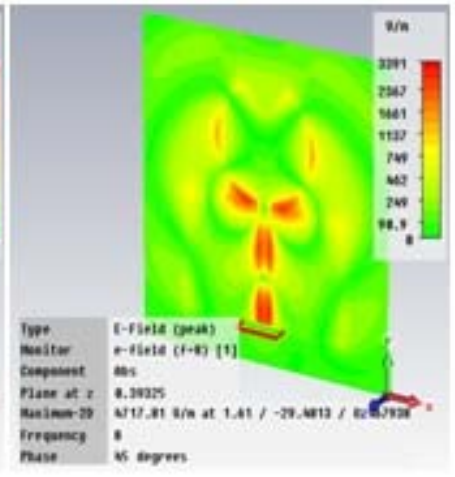

(c) $8 \mathrm{GHz}$

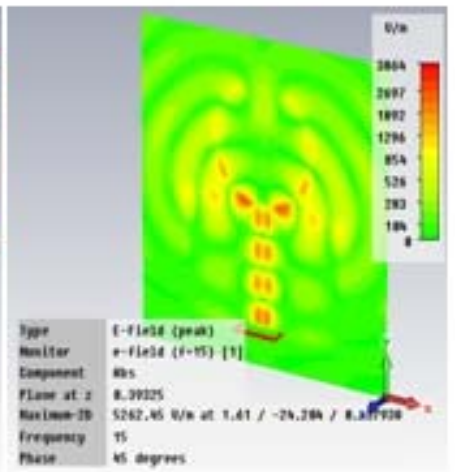

(f) $15 \mathrm{GHz}$

Figure 5 Current flow simulated results of the wearable textile UWB antenna design with six different selected frequency samples representing the overall achieved bandwidth 


\subsection{Simulated and Measured Radiation Pattern Results}

Although the limitation of measurement equipments used, the behaviour of $2 \mathrm{D}$ simulated and measured radiation pattern components of UWB antenna using flannel fabric as substrate material along with copper sheet as the conducting material is presented. A snapshot of measured radiation pattern environment is depicted in Figure 6 where the antenna is located in an anechoic chamber. Furthermore, Figure 7 clarifies the 2D simulated and measured co-polar radiation pattern components in the horizontal and vertical planes with three different frequency samples at $5 \mathrm{GHz}, 8 \mathrm{GHz}$, and $12 \mathrm{GHz}$ achieved by UWB antenna design using flannel fabric as substrate material, where measurement process has been conducted in air space. However, for the sake of better comparison, the measured radiation pattern results were plotted in the range between $-90^{\circ}$ and $+90^{\circ}$. Moreover, the magnitude of the radiation pattern has been normalized to simplify the viewing of measured results.

Figure 7 (a) and (b) represent the simulated horizontal and vertical planes co-polar radiation pattern components respectively of UWB antenna design using copper conducting sheet and flannel fabric. On the other hand, the measured copolar radiation pattern components of the fabricated antenna

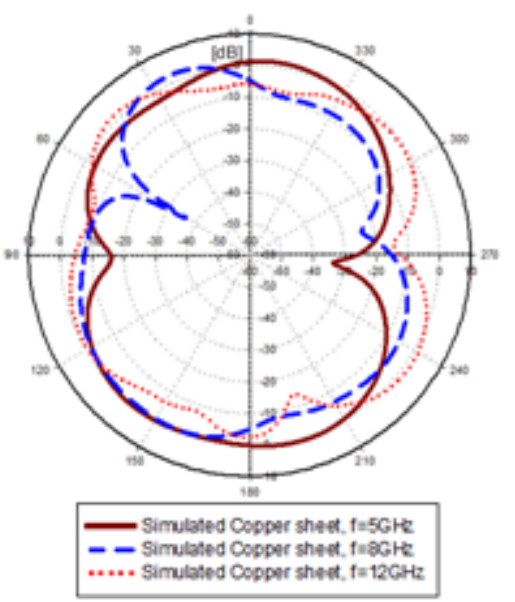

(a) E-Co Polar Horizontal plane of Simulated Copper sheet results

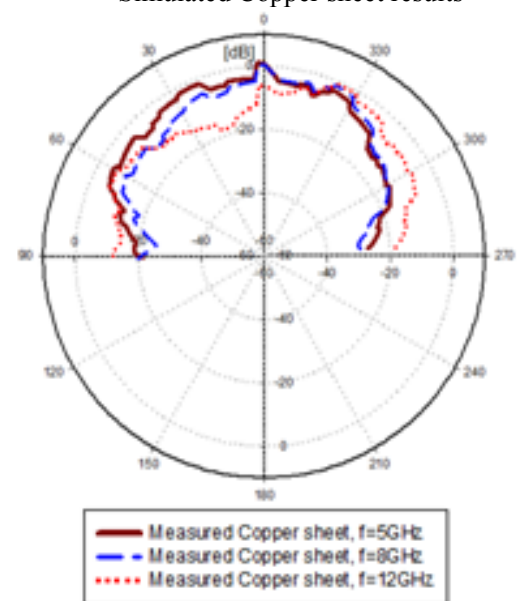

(c) E-Co Polar Horizontal plane of Measured Copper sheet results prototype using flannel fabric representing the horizontal and vertical planes respectively are demonstrated in Figure 7 (c) and (d).

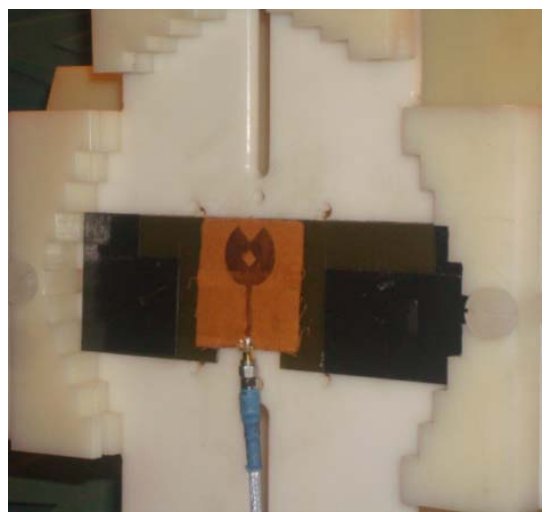

Figure 6 Snapshot of measured radiation pattern environment of the UWB textile antenna using flannel fabric as substrate material along with copper sheet as conducting material

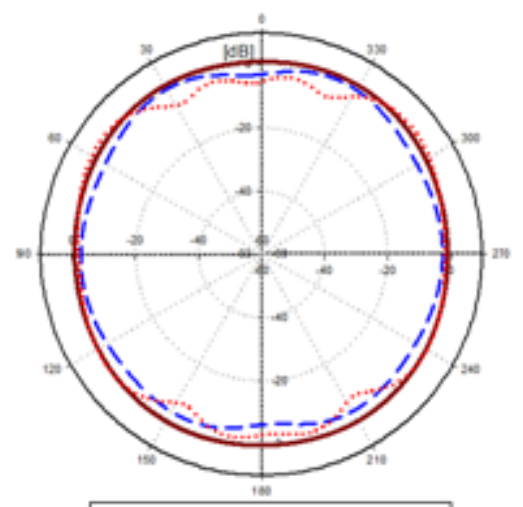

- - Simulated Copper sheet, $f=5 \mathrm{GHE}$ ..... Simulated Copper shent, f= $12 \mathrm{CHz}$

(b) E - Co Polar Vertical plane of Simulated Copper sheet results

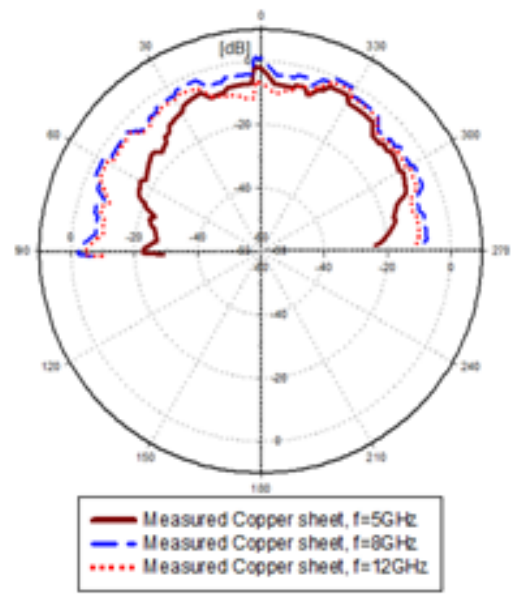

(d) E - Co Polar Vertical plane of Meaasured Copper sheet results

Figure 7 The behaviour of simulated and measured 2D radiation pattern results of the wearable textile UWB antenna design using flannel fabric substrate material considering three different selected frequency samples that represent the overall achieved bandwidth 
From Figure 7 (a) and (b), it can be noticed that the antenna resembles Omni-directional radiation pattern components in the vertical plane whatever the frequency under analysis, while two nulls are visible along the horizontal plane for each frequency under analysis. Moreover, from Figure 7, slight deviation between simulated and measured radiation pattern results in the horizontal and vertical planes can be observed. This difference can be related to fabrication tolerance and misalignment during measurement setup. However, both results correlate well to each other hence the patterns are similar. Results claimed that future antenna designers need to ensure that wearable telecommunication devices operate properly in the vicinity of human body. In particular, even though UWB antennas known by its low-power operation and extremely low radiated power, special attention must be paid to the Specific Absorption Rate (SAR) in order to avoid harm to human body.

\subsection{Simulated Gain anf Efficiency Results}

The variations of frequencies versus the gain of the wearable textile UWB antenna design are all demonstrated in Table 1 . The maximum gain achieved was about $4.8 \mathrm{~dB}$ at $10 \mathrm{GHz}$, while the lowest gain achieved is almost $2.6 \mathrm{~dB}$ at $2 \mathrm{GHz}$. However, the highest percentage of efficiency reached $95 \%$ at $6 \mathrm{GHz}$ and 9 $\mathrm{GHz}$, while the lowest efficiency was about $85 \%$ at $15 \mathrm{GHz}$. Consequently, results showed that the current antenna design has low power consumption due to the achievement of gain results that was less than $5 \mathrm{~dB}$ in most range of frequencies between 2 $\mathrm{GHz}$ and $15 \mathrm{GHz}$. In addition, the efficiency of the antenna could be considered vast compared to commercial antennas.

Table 1 Gain and efficiency simulated results of the textile UWB antenna design using flannel fabric as substrate material

\begin{tabular}{ccc}
\hline $\begin{array}{c}\text { Frequency } \\
\text { [GHz] }\end{array}$ & $\begin{array}{c}\text { Gain } \\
\text { [dB] }\end{array}$ & $\begin{array}{c}\text { Efficiency } \\
{[\%]}\end{array}$ \\
\hline 2 & 2.6 & 92 \\
3 & 3.6 & 93 \\
4 & 3.0 & 93 \\
5 & 2.8 & 94 \\
6 & 3.4 & 95 \\
7 & 4.2 & 94 \\
8 & 4.0 & 92 \\
9 & 4.2 & 95 \\
10 & 4.8 & 91 \\
11 & 4.3 & 89 \\
12 & 3.9 & 91 \\
13 & 4.0 & 92 \\
14 & 4.0 & 91 \\
15 & 4.0 & 85 \\
\hline
\end{tabular}

\subsection{CONCLUSION}

UWB antennas with partial ground plane are designed, fabricated and measured. The substrate of the designed antennas was made from two different types of substrate fabric materials (flannel and jeans) while the radiating element and ground plane were made from copper self-adhesive sheet. The operating frequency of simulated and measured results of the current manuscript designs span from $2 \mathrm{GHz}$ up to $15 \mathrm{GHz}$. $\left(\left|\mathrm{S}_{11}\right|\right)$ results indicated that flannel and jeans fabrics acted and performed as good candidate for textile wearable applications, and hence raised issues and possibilities of replacing rigid board substrate materials. Results further indicated that the measured dielectric properties values of both fabrics were valid enough to provide design information, since the targeted UWB frequency range is reasonably well met and hence produced comparable measured results. Moreover, results showed that the current antenna design has low power consumption due to the achievement of gain results that was less than $5 \mathrm{~dB}$ in most range of frequencies between $3 \mathrm{GHz}$ and 15 GHz. In addition, the efficiency of the antenna could be considered vast when compared with commercial antennas. Results claimed that future antenna designers need to ensure that wearable telecommunication devices operate properly in the vicinity of human body. In particular, special attention must be paid to the Specific Absorption Rate (SAR) in order to avoid harm to human body.

\section{Acknowledgement}

Authors of the current manuscript wish to acknowledge the support of Ministry of Higher Education (MOHE), Research Management Centre (RMC), Radio Communication Engineering Department (RaCED) Faculty of Electrical Engineering, Universiti Teknologi Malaysia for supporting this research work under grant No.: Q.J130000.7123.02H02 and 74578. Authors also wish to thank Sudan University of Science and Technology for supporting this research work.

\section{References}

(1) Ben, A., M. Dohler, E. E. Okon, W. Q. Malik , A. K. Brown and D. J. Edwards. 2007. Ultra-wideband Antennas and Propagation for Communications, Radar and Imaging. Wiley

(2) M. A. R. Osman, M. K. A. Rahim, M. Azfar. A, K. Kamardin, F. Zubir, and N. A. Samsuri. 2011. Performance and Evaluation of UltraWideband Wearable Textile Antenna. IEEE European Conference on Antennas and Propagation (EuCAP). April 11-15. Rome, Italy.

(3) B. Sanz-Izquierdo, J. C, Batchelor and M. I. Sobhy. Compact UWB Wearable Antenna. Department of Kent, The University of Kent, Canterbury, Kent.

(4) Faranak, N. 2005. Ultra-wideband Communications: Fundamentals and Applications. Prentice Hall.

(5) Jonna, P. 2001. Antenna Design for Ultra Wideband Radio. Electrical Engineering, New Mexico State University.

(6) M. Tanaka, J. H. Jang. 2003. Wearable Microstrip Antenna. 2003 IEEE AP-S International Symposium on Antennas and Propagation and URSI North American Radio Science Meeting, Columbus, OH, USA, June.

(7) K. Fujimoto and J. R. James. 2001. Mobile Antenna System Handbook. Second Edition. Artech House, INC., Norwood, MA.

(8) P. Salonen, H. Hurme. 2003. A Novel Fabric WLAN Antenna for Wearable Applications. 2003 IEEE AP-S International Symposium on Antennas and Propagation and URSI, North American Radio Science Meeting, Columbus, OH, USA, June.

(9) Lukasz Januszkiewicz, Slawomir Hausman, Tomasz Kacprzak. 2007. Textile Body-Worn Exponentially Tapered Vee Antenna. Electronic Letters. 42(3).

(10) A. Tronquo et al. 2006. Robust Planar Textile Antenna For Wireless Body Lans Operating In 2.45ghz ISM Band. Electronic Letters. 42 (3).

(11) B. Sanz-Izequierdo, F. Huang and J.C. Batchelor. Dual Band Button Antenna for Wearable Applications. Department of Electronics, The University of Kent, Canterbury, Kent.

(12) R. Chandran and W. G. Scanlon. 2010. Dual-band low profile antennas for body-centric communications. International Workshop on Antenna Technology (iWAT).1-3 March Lisbon, Portugal. 1-4.

(13) R. L.Burden, J. D. Faires, and A. C. Reynolds. 1978. Numerical Analysis, Boston, Prindle Weber and Schmidt. 31-38.

(14) A. G. Derneryd. 1979. Analysis of the microstrip disk antenna element IEEE Transactions on Antennas and Propagation, September. 27(5): $660-664$. 
(15) K. Hirasawa, and M. eds. Haneishi. 1992. Analysis Design and Measurement of Small and Low-Profile Antennas. London, Artech House. 69.

(16) C. A. Balanis. 2004. Antenna Theory: Analysis snd Design. NewYork: John Wiley and Sons
(17) W. L. Stutzman and G.A. Thiele. 1998. Antenna Theory and Design. Second Edition, John Wiley \& Sons, New York. 